\title{
MISCELLANÉES
}

\section{PROBLEME $N^{\circ} 8$}

Un architecte naval parmi les plus conscien. cieux, calcule le poids du nouveau navire qui, si tout va bien, doit être demain lorgueil de toute sa carrière.

Depuis le fin rond de la cale jusqu'au sommet des cheminées, il dé aille les pièces une à une, sans omellre une poignée de porte ou un rivet.

Puis, après s'ètre entouré de toutes les précautions possibles, il exécute des mesures des inées à déterminer la clensité exacte de leau du bassin dans lequel doit ètre réceptionné son navire.

En possession de ces deux indications, il applique à la lettre le fameux principe dont le iexte signé «Archimede» figure en letres d'or sur la page de garde de la Bible des Armateurs et, sùr de lui, il fait tracer sur la coque la fine ligne blanche qui doit, très exactement coïncider avec la ligne de flottaison du bàtiment.

De plus, tenant à présenter un devis d'armement rédigé dans toutes les règles de son art, il calcule minutieusemeni la valeur de ce fameux ( o - a) bien connu de tous les membres de sa corporation, qui est l'un des termes entrant dans lexpression du couple de redressement, luimème facteur essentiel de la stabilité du bâtiment.

Or, il résulte des épreuves imposées par la marine :

d'abord que le navire semble plus lourd qu'il était prévu;

- ensuite que le (o _ a) trouvé à la suite de l'épreuve de stabilité est plus faible que celui indiqué par le constructeur.

Celui-ci, apres avoir une lois de p'us repris tous ses calculs ne peut plus douter de leur exactilude et en vient à se demander si toutes les hypotheses habituelles qu'il a faites sont exactes et, en pariculier, s'il n'y a pas lieu de tenir compte du fait que toutes ces opérations ont lieu dans l'air atmosphérique et non dans le vide.

Est-ce vrai ou faux ?

Puis l'idée lui vient de vérifier par lui-mêmæ ce qu'affirme Archimède. Il construit, de ses propres mains, un petit navire destiné à croiser dans sa baignoire.
Pour déterminer le déplacement de ce joujou, il opère simplemeni de la lacon suivante : l'un des plateaux d une brance de précision recoit son ouvrage et une éprouvette identique dans laquelle il verse de l'eau de sa baignoire jusqu'à obtenir l'équilibre.

Par une opération précédente, l'égalité de poids des deux éprouveltes avai écé vériliée.

Dans la suite de ces mesures, notre ami part du principe que le volume deau déplacé par son navire est rigoureusement égal à celui qu'il a versé dans son éprouvette. Est-ce rat ou faux?

Disons seulement, en lerminant, que de lelles subtilités ont fait l'obję, an début de ce siècie, d'une controverse tres anmée au sein du Corps des Ingénieurs de notre Marine.

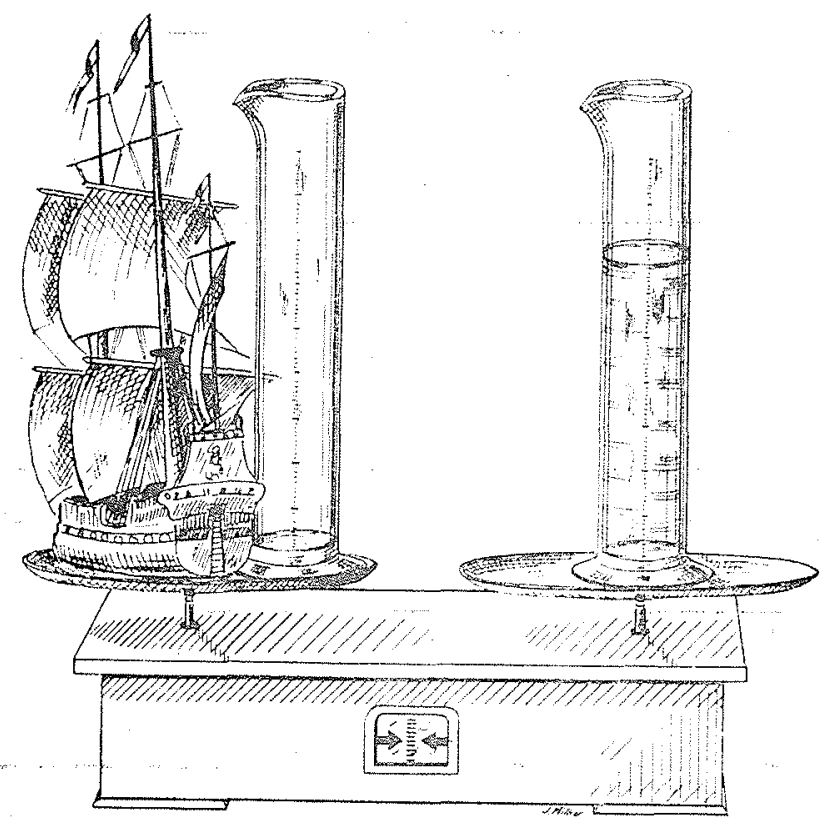

PROBLENE N"9

Dans le pare d'at ractions populaire de Copenhaģue, dénommé «Le Tivoli », il est une maison magique. Et dans lune des sal'es de cette maison, il est une pompe enchantée, attirante pour Jes visileurs qui ne manquent pas de l'actionner.

Mais alors, a lenr grande surprise, l'eau sort du pilier creax $C$ el apparemment remonte l'auge de $B$ vers $A$. Bien qu'il s'agisse la d'une technique voisine de l'illusionnisme, un de nos corres- 
pondants nous prie de soumettre la question s la sagacité de nos lecteurs et de leur demander yils connaissent des dispositifs analogues en d'atutres pays. Tous les commentaires seront les bienvenus.

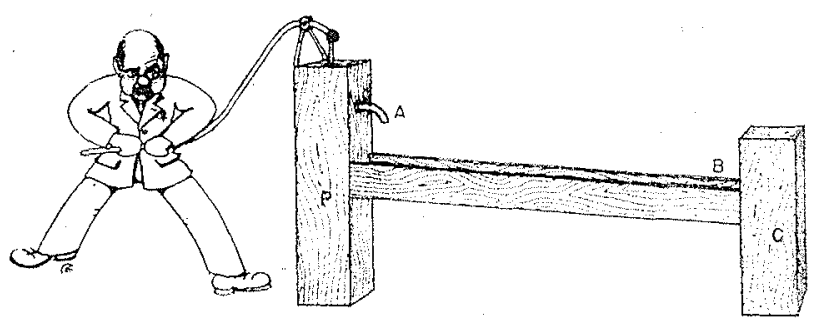

\section{PROBLEME N"10}

La littérature offre parfois de surprenantes curiosités à l'hydraulicien, La fable de La Fonlaine que nous reproduisons ci-dessous constitue une amusante illustration de cette affirmation.

Nous demandons à nos lecteurs de nous faire connaitre leurs réactions d'hydraulicien vis-àvis de cette fable, mais nous leur recommandons loutefois de temperer leur jugement par la forme bégante de leur réponse, de façon que le s bonhomme $»$, s'il vivait encore, n'en fusse point oftusqué.

\section{« L'ANE CHARGE D'EPONGES \\ E'T L'ANE CHARGE DE SEL »}

Un Anier, son sceptre à la main,

Menoit, en empereur romain,

Deux Coursiers, a longues oreilles.

Liun d'éponges chargé, marchoit comme un [courrier :

Et lautre, se faisant prier,

Portoil, comme on dit, les bouteilles:

Sa charge était de sel. Nos gaillards pèlerins,

Par monts, par vaux, et par chemins,

Au gué d'une rivière à la fin arrivèent,

Et fort empechés se trouverent.

L'Anier, qui tous les jours traversoit ce gué-la,

Sur l'Ane à l'éponge monta,

Chassant devant lui l'autre bète,

Qui roulant en faire à sa tête,

Dans un trou se précipita,

Revint sur l'eau, puis échappa,

Cin au bout de quelques nagées,

Toul son sel se fondit si bien

Que le Bandet ne sentit rien

Sur ses epaules soulagées.

Camarade epongier prit exemple sur lui,

Comme un mouton qui va dessus la foi d'autrui.

Voila mon Ane a l'eau; juscu'au col il se plonge.

Lui, le conducteur et l'éponge.

Tous trois burent d'autant : l'Anier et le Grison
Firent à l'éponge raison.

Celle-ci devint si pesante,

Et de tant d'eau s'emplit d'abord,

Que l'Ane succombant ne put gagner le bord,

L'Anier l'embrassoit, dans l'attente

D'une prompte et certaine mort.

Quelqu'un vint an secours: qui ce fut, il

n'importe:

C'est assez qu'on ait vu par là qu'il ne faut point Agir chacun de même sorte.

J'en voulais venir à ce point.

\section{REPONSE AUX PROBLEMES}

Nous donnons, aujourd'hui, la réponse que M. Darrases a bien voulu nous communiquer; nous pensons que le texte de l'éminent Ingénieu: en chef de la Compagnie Electromécanique constitue la meilleure conclusion aux différentes discussions soulevées par cette question et nous l'en remercions rivement.

Dans sa lettre que nous reproduisons intégralement, le distingué Académicien nous apporte également une réponse au problème de l'œuf' sur le jet d'eau; la solution présentée coüncide aveo celle donnée par $O$. Reynouds, dont nous donnerons dans notre prochain numéro, le texte ; par la mème occasion, nous exposerons les résultats que nous avons trouves en refaisant les propres expériences de Reynords.

\section{Probleme de l'Ecluse}

« L'intéressant problème soulevé par Simon Gritio me paraît appeler les réflexions suivantes:

L'affirmation de la possibilité de principe d'une remontée d'eau par l'éclusée de bateanx plus chargés à la descente qu'à la remontée, est certainement juste, comme le confirme M. Dupis dans rotre numéro de Mai, et si la démonstrafion un pen sommaire de Grapio n'a pas entrainć une adhésion unanime et immédiate, landis que MrNaro lui substituait un raisonnement inutilement compliqué et confus pour aboutir, en définitive, a des conclusions erronées, e'est que ni I'un ni l'autre n'ont pris la précaution élémentaire de considérer un cycle fermé d'opérations. Ce cycle fermé doit nécessairement comprendre la charge et la decharge des bateaux, qui en faisant varier leur tirant d'eau, influent naturellement sur le plan d'eau des biefs. Si nous. supposons, pour fixer les idées, que les niveaux dean des deux biefs soient maintenus constants par communication respective avec deux réservoits indéfinis, il devient évident que toul échange d'un bateau vide de déplacement st contre un bateau chargé de déplacement St2 entrainera la remontee d'un volume d'eau correspondant it 
la différence et dont il $y$ a lieu seulement de retrancher la perte $\mathrm{Sx}$ de l'éclusée.

La considération du point de vue énergétique confirme cette conclusion.

En effet, tandis que le travail « non compensé » (au sens themodynamique) ou perte $2 \frac{1}{2} g$ o S $x$ qu'entrainent la vidange, puis le remplissage du sas, est du 2 ordre en $x$ (hauteur de l'éclusée), le travail net de la pesanteur pour l'échange d'un bateau chargé descendant contre un bateau vide remontant est go:S $\left(t_{2}-t_{1}\right) x$, cest-à-dire du $1^{\text {er }}$ ordre en $x$.

Le rapport de la perte au travail développé peut donc être rendu aussi petit qu'on le veut par une réduction convenable de $x$, et la différence, qui doit se retrouver quelque part, consiste précisément en le travail utile d'élévation du volume d'eau $S\left(t_{2}-t_{1}\right)$.

L'apport de ce volume d'eầu dans le bief supérieur n'aurait pas échappé à l'attention de Mrnard s'il avait pris en considération, pour compléter son cycle, la phase de rechargement des bateaux vides, qui comporte évidemment une augmentation de leur déplacement et fait ainsi apparaître en excédent le volume correspondant.

Le problime devient particulièrement clair, si l'on imagine que le chargement des bateaux ait pour densité moyenne celle même de l'eau, ou consiste par exemple en eau simplement colorée pour la distinguer de l'eau ordinaire ; auquel cas, le transport revient à un simple echange de l'eau colorée descendante contre un volume égal d'eau ordinaire remontante, au prix d'une perte de volume $\mathrm{S} x$ due à l'éclusée qui, proportionnelle à $x$, peut être rendue aussi petite qu'on le veut par une multiplication convenable du nombre de biefs.

\section{Probleme de l'Cuf}

Le maintien en équilibre d'une boule légère sur' un jet d'eau s'explique par la composante horizontale de la réaction que détermine sur la bande, roulant autour d'un axe horizontal, Ia projection de gouttelettes dans la direction même suivant laquelle le centre de la boule tend à s'écarter de l'axe du jet.

Celte réaction se transmet à la boule gràce à l'adhérence que présente, avec elle, par capillarité, un bourrelet d'eau qui, l'enveloppant plus ou moins complètement suivant un arc de grand cercle, alimente les projections de gouttes qu: s'en délachent suivant la tangente. C'est la prépondérance vers le haut et vers l'axe du jet, de la force centrifuge s'exerçant dans ce bourrelet qui empèche la boule de s'écarter du jet et de romber.

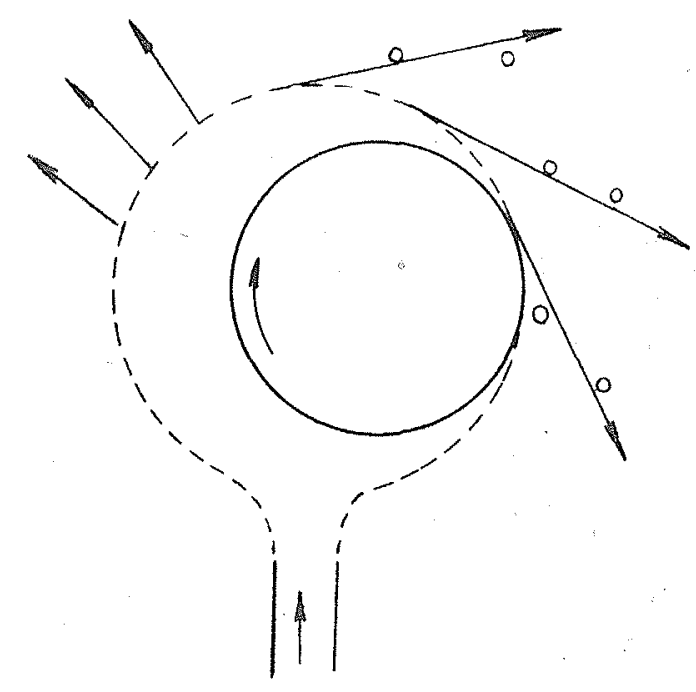

L'efret disparaît naturellement si le liquide cesse de mouiller la boule, soit que cellemci ail èté graissée, soit que l'eau soit remplaće par du mercure dont un jet doit se montrer inapte à mainienir en équilibre un cuf ou une boule. comme le font par contre le jet d'eau ou mème - pour des raisons analogues quoique un peu diflérentes - un jet d'air.

J'ai entendu dire, mais sans avoir eu l'occasion de le vérifier, que l'explication ci-dessus aurait été fournie par O. REYNouds.

J'espère que les remarques ci-dessus vous paraitront intéressantes et, permettez-moi à cette occasion de vous féliciter d'avoir pris l'iniliative de proposer ainsi à vos lecteurs cette sorte de problèmes que je souhaite voir bien accueillis comme ils le méritent car, si leur intérêt pratique peut parâtre nul à première vue, ils sont particulièrement propres à susciter un esprit critique utile, ainsi que les facultés d'attention, d'intuition, d'imagination et enfin le sens physique des phénomènes que notre enseignement, trop souvent purement formel, tend quelque peu à négliger. "

\section{G. Darrieus.}

